\title{
As habilidades socioemocionais necessárias ao enfrentamento da COVID-19
}

The socioemotional skills necessary to cope with COVID-19

Las habilidades socioemocionales necesarias para hacer frente a COVID-19

DESCRITORES: Saúde Mental; Pandemia; COVID-19.

DESCRIPTORS: Mental Health; Pandemic; COVID-19.

DESCRIPTORES: Salud Mental; Pandemia; COVID-19.

RECEBIDO EM: 20/05/2020 APROVADO EM: 20/05/2020

\section{Bruno da Silva Lourenço}

Enfermeiro. Mestre em Enfermagem em Saúde Mental. Especialista em Enfermagem em Terapia Intensiva pela EEAN/UFRJ.

Professor Auxiliar da Universidade Estácio de Sá. https://orcid.org/0000-0002-8977-1842

\section{Janaina Rosa Lourenço da Silva}

Enfermeira. Especialista em Enfermagem em Cuidados Intensivos com Ênfase no Cliente Adulto e Idoso pela UFF e em Enfermagem Neonatal pela UGF. https://orcid.org/0000-0001-8391-8471

\section{Niquélen Bianca Miller França}

Enfermeira. Doutora em Psicologia Social. Mestre em Psicologia pela Universidad John Kennedy. Professora Auxiliar da Universidade Estácio de Sá.https://orcid.org/0000-0002-9816-5465

\section{Teresinha Herdvig Praun}

Enfermeira Obstetra. Especialista em Pedagogia Aplicada à Saúde. Mestranda em Educação pela FUNıBER. Professora Auxiliar da Universidade Estácio de Sá. https://orcid.org/0000-0002-0316-1876

\section{Kenia Oliveira Barbosa da Hora}

Enfermeira. Mestranda em Enfermagem pela EEAN/UFRJ. Especialista em Terapia Intensiva e Oncologia. Professora Auxiliar da Universidade Estácio de Sá. https://orcid.org/0000-0002-2887-7408

\section{Lidiane Dias Reis}

Enfermeira. Mestre em Enfermagem. Especialista em Gestão do Trabalho e Educação em Saúde e em Saúde da Família. MBA em Gestão em Saúde. Professora Auxiliar da Universidade Estácio de Sá. https://orcid.org/0000-0001-6577-6545

\section{Cristiano Saldanha Silva}

Enfermeiro. Especialista em Emergências Médicas, Pacientes Críticos e Centro Cirúrgico. Mestrando em Saúde da Família pela Estácio de Sá. Professor Auxiliar da Universidade Estácio de Sá. https://orcid.org/0000-0003-3847-4925

A COVID-19 foi inicialmente detectada na cidade de Wuhan, capital da província da China Central, em dezembro de 2019. Seu nome advém da síndrome respiratória ocasionada pelo novo coronavírus. A Organização Mundial da Saúde (OMS) declarou, em
30 de janeiro de 2020, que o surto da doença causada constitui uma Emergência de Saúde Pública de Importância Internacional - o mais alto nível de alerta da Organização, conforme previsto no Regulamento Sanitário Internacional. Em 11 de março de 2020, a COVID-19 foi caracterizada pela OMS como uma pandemia. A facilidade de propagação, a falta de conhecimento sobre o vírus e o aumento exponencial do número de contágios colaborou para isso ${ }^{(1)}$.

Até o dia 18 de maio de 2020 foram confirmados no mundo $4.618 .821 \mathrm{ca}$ - 
sos de COVID-19 e 311.847 mortes $^{(2)}$. Conforme as informações atualmente disponíveis, a via de transmissão de uma pessoa a outra do SARS-CoV-2 ocorre por meio de gotículas respiratórias (expelidas durante a fala, tosse ou espirro) e também pelo contato direto com pessoas infectadas ou indireto por meio das mãos, objetos ou superfícies contaminadas, de forma semelhantes com que outros patógenos respiratórios se disseminam. Além disso, tem-se estudado a possibilidade de transmissão do vírus por meio de aerossóis (partículas menores e mais leves que as gotículas) gerados durante manipulação direta da via aérea, como na intubação orotraqueal ou em outros procedimentos potencialmente geradores de aerossóis ${ }^{(3)}$.

Não há um nível de complexidade comum a todos, sendo os casos mais graves acometidos de uma insuficiência respiratória aguda que requer cuidados hospitalares intensivos, com uso de ventilação mecânica, em algumas situações ${ }^{(4)}$. Os sintomas mais comuns dessas infecções podem incluir manifestações respiratórias (tosse, dificuldade para respirar, batimento das asas nasais, entre outros) e febre, cuja qual pode não estar presente em alguns pacientes, como crianças, idosos, imunossuprimidos ou que tomam medicamentos para diminuir a febre ${ }^{(3)}$.

Com o objetivo de reduzir os impactos da pandemia, diminuindo o pico de incidência e o número de mortes, alguns países têm adotado medidas como o isolamento de casos suspeitos, fechamento de escolas e universidades, distanciamento social de idosos e outros grupos de risco, bem como quarentena de toda a população. Estima-se que essas medidas cheguem a alcançar o "achatamento da curva" de infecção, ao favorecer um menor pico de incidência em um dado período, reduzindo as chances de que a capacidade de leitos hospitalares, respiradores e outros suprimentos seja insuficiente frente ao aumento repentino da demanda, o que se poderia ocasionar uma maior mortalidade ${ }^{(5)}$.

\section{A SAÚDE MENTAL NO CONTEXTO DE PANDEMIA}

Estudos têm sugerido que o medo de ser infectado por um vírus potencialmente fatal, de rápida disseminação, cujas origens, natureza e curso ainda são pouco conhecidos, acaba por afetar o bem-estar psicológico de muitas pessoas ${ }^{(6)}$.

Na vigência de pandemias, a saúde física das pessoas e o combate ao agente patogênico são os focos primários de atenção de gestores e profissionais da saúde, de modo que as implicações sobre a saúde mental tendem a ser negligenciadas ou subestimadas. Sendo assim, discutir questões que norteiam a preservação desse componente mental pode ser de grande importância, para que o adoecimento psíquico não gere impactos maiores do que a própria $\mathrm{CO}$ VID-19, gerando prejuízos maiores em diferentes segmentos da sociedade ${ }^{(7)}$.

Durante uma pandemia, é esperado que boa parte da população esteja em estado de alerta, preocupados, confusos, estressados e com sensação de falta de controle frente às incertezas do momento. Com isso, pode ser previsível que uma parte dessa mesma população exposta possa vir a sofrer alguma manifestação psicopatológica, caso não seja feita nenhuma intervenção de cuidado específico para as reações e sintomas manifestados. Os fatores que influenciam o impacto psicossocial estão relacionados ao que cerca a pandemia e o grau de vulnerabilidade em que a pessoa se encontra naquele dado momento. Entretanto, é importante destacar que nem todos os problemas psicológicos e sociais apresentados poderão ser qualificados como doenças. A maioria será classificado como reações normais diante de uma situação anormal.

A COVID-19 impacta os seres humanos de maneiras gerais e específicas, em função de uma série de fatores e determinantes, a começar da necessidade de se adaptar aos novos protocolos de biossegurança, da desconfiança na gestão e coordenação dos protocolos de biossegurança por autoridades, do risco de ser infectado e infectar outros, das fa- mílias que se separam em função da atividade laboral em situações de linha de frente de combate ao novo coronavírus, da percepção equivocada de que sintomas que podem estar atrelados a outros problemas de saúde (como dor de cabeça, espirros e febre, por exemplo) podem ser confundidos com a COVID-19, da preocupação por seus filhos ficarem sem as referências de cuidado e trocas sociais, isto é, sem a convivência nas escolas, universidades, dos encontros de amigos e grande distanciamento da sua rede socioafetiva (avós, amigos, vizinhos etc) e do risco de agravamento de saúde mental e física de crianças, pessoas com deficiência ou idosos que tenham sido separados de seus pais ou cuidadores devido à quarentena. São motivos suficientes para impactar a saúde mental.

Em uma revisão de literatura sobre a quarentena, foi identificado que os efeitos negativos dessa medida incluem sintomas de estresse pós-traumático, confusão e rai$\mathrm{va}^{(8)}$. Um estudo com a população geral na China, incluindo 1.210 participantes em 194 cidades, durante o estágio inicial da pandemia, demonstrou os impactos na saúde mental diante da pandemia do novo coronavírus. Esse estudo revelou sintomas moderados a severos de ansiedade, depressão e estresse, em $28,8 \%, 16,5 \%$ e $8,1 \%$ dos respondentes, respectivamente. Além disso, $75,2 \%$ dos respondentes referiram medo de que seus familiares contraíssem a doença. Ser mulher, estudante e apresentar sintomas físicos ligados à COVID-19, ou problemas de saúde prévios, foram fatores significativamente associados a maiores níveis de ansiedade, depressão e estresse. Em contraponto, receber informações precisas sobre situação local da doença, formas de prevenção e de tratamento, consistiram em fatores significativamente associados a menores níveis de ansiedade, depressão e estresse ${ }^{(9)}$.

\section{HABILIDADES SOCIOEMOCIONAIS NA PANDEMIA}

Com passar do tempo e com o avanço do vírus ceifando milhões de vidas hu- 
manas, nas pessoas afastadas dos familiares e amigos, começam a emergir os sentimentos de impotência, insegurança, medo, angústia e de ansiedade diante dessa ameaça invisível. Neste sentido, as habilidades socioemocionais, descritas como uma estrutura cognitiva e comportamental capaz de englobar suas capacidades de controlar suas emoções diante das situações e pessoas, para uma existência com responsabilidade, de forma livre e para uma vida com realização de valores, devem ser empregadas no cotidiano de todos ${ }^{(10)}$.

As habilidades socioemocionais são um agrupamento de comportamentos realizado por uma pessoa em um contexto sociocultural, de forma que esta possa expor os seus sentimentos, atitudes, desejos, opiniões de maneira não ofen- siva aos demais componentes do grupo, com a finalidade de minimizar conflitos e resolver problemas com uma conduta assertiva ${ }^{(11)}$. O contexto vivido por profissionais na linha de frente e pela sociedade é fortemente impactado pelas transformações que o mundo vive nesse momento. Desempenhar essas habilidades em equilíbrio pode ser importante aliada no enfrentamento dos desafios que se apresentam.

Em suma, o desenvolvimento das habilidades socioemocionais para uma existência realizada, necessita de uma base epistemológica da educação para aprender a ser e aprender o dever ser num mundo globalizado, em prol da promoção da saúde e da prevenção de comorbidades, de forma responsável com atitudes de ressignificação e transformação para o enfrentamento da COVID-19. Uma vez que a pessoa decide, por comportamentos e hábitos saudáveis, se poderá suportar os reveses da vida de maneira equilibrada em qualquer situação sociocultural ${ }^{(12)}$.

Desta forma, diante do fenômeno do novo coronavírus, que emergiu a crise pandêmica mundial, o medo e a insegurança têm dominado os sentimentos e sensações de todos, principalmente pela falta de habilidade em superar esta questão e ainda por não saber por quanto tempo essa situação de perdurará. No entanto, essas circunstâncias ainda imutáveis, não serão eternas, e podem ser utilizadas para uma transformação em novas oportunidade para clarificar e realizar os valores, as crenças, um reaprender o convívio em família, o cuidado com outro e consigo mesmo.

\section{REFERÊNCIAS}

1. World Health Organization. Coronavirus disease 2019 (COVID-19) Situation Report 78 [Internet]. 2020 [acesso em 05 mai 2020]. Disponivel em: http://www.who.int/docs/default-source/ coronaviruse/situation-reports/20200407-sitrep-78-covid-19. pdf?sfursn=bc43e1b_2.

2. Organização Pan-Americana da Saúde. Folha informativa COVID-19 (doença causada pelo novo coronavírus) [Internet]. Brasília (DF); OPAS, 2003 [acesso em 05 mai 2020]. Disponivel em: https://www.paho.org/bra/index.php?option=com_content\&view=article\&id=6101: covid19\&Itemid=875.

3. Agência Nacional de Vigilância Sanitária. Nota Técnica GVIMS/ GGTES/ANVISA n. ${ }^{\circ}$ 07/2020 - Orientações para a prevenção da transmissão de covid-19 dentro dos serviços de saúde [Internet]. 2020 [acesso em 05 mai 2020]. Disponível em: https:// www20.anvisa.gov.br/segurancadopaciente/index.php/alertas/ item/nota-tecnica-gvims-ggtes-anvisa-n-07-2020?category_ id $=244$.

4. Centers for Disease Control Prevention. Severe outcomes among patients with Coronavirus Disease 2019 (COVID-19) - United States, February 12-March 16, 2020. Morbidity and Mortality Weekly Report [Internet]. 2020; 69(12):343-346. DOI: http://doi.org/10.15585/mmwr.mm6912e2.

5. Ferguson N, Laydon D, Nedjati Gilani G, Imai N, Ainslie K, Baguelin M, Ghani A. Report 9: Impact of non-pharmaceutical interventions (NPIs) to reduce COVID19 mortality and healthcare demand [Internet]. 2020. DOI: https://doi.org/10.25561/77482.

6. Asmundson GJG, Taylor S. Coronaphobia: Fear and the 2019nCoV outbreak. Journal of Anxiety Disorders [Internet]. 2020; 70:102196. DOI: https://doi.org/10.1016/j.janxdis.2020.102196.
7. Ornell F, Schuch JB, Sordi AO, Kessler FHP. "Pandemic fear" and COVID-19: Mental health burden and strategies. Brazilian Journal of Psychiatry [Internet]. 2020 [acesso em 10 mai 2020]. Disponível em: https://www.rbppsychiatry.org.br/details/943/ en-US/-pandemic-fear--and-covid-19--mental-health-burden-and-strategies.

8. Brooks SK, Webster RK, Smith LE, Woodland L, Wessely S, Greenberg N, Rubin GJ. The psychological impact of quarantine and how to reduce it: Rapid review of the evidence. The Lancet [Internet]. 2020; 395(10227):912-920. DOI: https://doi.org/10.1016/ S0140-6736(20)30460-8.

9. Wang C, Pan R, Wan X, Tan Y, Xu L, Ho CS, Ho RC. Immediate psychological responses and associated factors during the initial stage of the 2019 coronavirus disease (COVID-19) epidemic among the general population in china. International Journal of Environmental Research and Public Health [Internet]. 2020; 17(5):1729. DOI: https://doi.org/10.3390/ijerph17051729.

10. Terradas SA. Habilidades para una Vida con Sentido. Argentina: Escuela de Vida; 2011.

11. Turini BSA, Kester C. Habilidades sociais e análise do comportamento: compatibilidades e dissensões conceitual-metodológicas. Psicol. rev. [online] [Internet]. 2010 [acesso em 18 mai 2020]; 16(2):330-350. Disponivel em: http://pepsic.bvsalud.org/ pdf/per/v16n2/v16n2a07.pdf.

12. Aed ALZ. O Desenvolvimento das habilidades socioemocionais como caminho para a aprendizagem e o sucesso escolar de alunos da educação básica. Constr. psicopedag. [Internet]. 2016 [acesso em 17 mai 2020]; 24(25). Disponível em: http://pepsic.bvsalud.org/scielo.php?script=sci_arttext\&pi$d=S 1415-69542016000100002$. 